\title{
A Novel Tetrapeptide Derivative Exhibits In Vitro Inhibition of Neutrophil-Derived Reactive Oxygen Species and Lysosomal Enzymes Release
}

\author{
Sumitra Miriyala, ${ }^{1,2}$ Manikandan Panchatcharam, \\ Meera Ramanujam, ${ }^{1,3}$ and Rengarajulu Puvanakrishnan ${ }^{1}$ \\ ${ }^{1}$ Department of Biotechnology, Central Leather Research Institute, Chennai 600020, India \\ ${ }^{2}$ Department of Cellular Biology and Anatomy, Louisiana Health Sciences Center, Shreveport, LA 71130, USA \\ ${ }^{3}$ Immunology and Inflammation, Boehringer Ingelheim Pharmaceuticals, Inc., Ridgefield, CT 06877, USA
}

Correspondence should be addressed to Sumitra Miriyala; smiriy@lsuhsc.edu

Received 28 February 2013; Revised 17 April 2013; Accepted 18 April 2013

Academic Editor: Aimee Landar

Copyright (C) 2013 Sumitra Miriyala et al. This is an open access article distributed under the Creative Commons Attribution License, which permits unrestricted use, distribution, and reproduction in any medium, provided the original work is properly cited.

\begin{abstract}
Neutrophil infiltration plays a major role in the pathogenesis of myocardial injury. Oxidative injury is suggested to be a central mechanism of the cellular damage after acute myocardial infarction. This study is pertained to the prognostic role of a tetrapeptide derivative PEP1261 (BOC-Lys(BOC)-Arg-Asp-Ser(tBu)-OtBU), a peptide sequence (39-42) of lactoferrin, studied in the modulation of neutrophil functions in vitro by measuring the reactive oxygen species (ROS) generation, lysosomal enzymes release, and enhanced expression of $\mathrm{C}$ proteins. The groundwork experimentation was concerned with the isolation of neutrophils from the normal and acute myocardial infarct rats to find out the efficacy of PEP1261 in the presence of a powerful neutrophil stimulant, phorbol 12-myristate 13 acetate (PMA). Stimulation of neutrophils with PMA resulted in an oxidative burst of superoxide anion and enhanced release of lysosomal enzymes and expression of complement proteins. The present study further demonstrated that the free radicals increase the complement factors in the neutrophils confirming the role of ROS. PEP1261 treatment significantly reduced the levels of superoxide anion and inhibited the release of lysosomal enzymes in the stimulated control and infarct rat neutrophils. This study demonstrated that PEP1261 significantly inhibited the effect on the ROS generation as well as the mRNA synthesis and expression of the complement factors in neutrophils isolated from infarct heart.
\end{abstract}

\section{Introduction}

Reactive oxygen species (ROSs) have been shown to exert a direct inhibitory effect on myocardial function in vivo and have a critical role in the pathogenesis of myocardial stunning $[1,2]$. Oxidative stress and formation of ROS could set off a cascade of biochemical and molecular squeal such as the xanthine dehydrogenase/xanthine oxidase conversion, leading to over production of ROS [3,4]. Oxidative ischemic injury is suggested to be a central mechanism of the cellular damage affecting all organs and tissues after ischemia; however, the mechanisms, which trigger and modulate this damage, have not been fully characterized.
Polymorphonuclear leukocytes (PMNLs) are short-lived, terminally differentiated cells that act against all infections and they are one of the most important cellular components involved in host defense. Circulating PMNLs participate in host defense by margination and extravasations at the site of inflammation [5]. Although neutrophils are essential to host defense, they have also been implicated in the pathology of ischemia $[6,7]$ and in many chronic inflammatory conditions $[8,9]$. Neutrophil levels are activated in myocardial infarction [10], and subsequently, activated neutrophils produce reactive oxygen species (ROS) such as superoxide anion $\left(\mathrm{O}_{2}{ }^{--}\right)$, hydrogen peroxide $\left(\mathrm{H}_{2} \mathrm{O}_{2}\right)$, hypochlorous acid $(\mathrm{HOCl})$, and possibly hydroxy radical $\left(\mathrm{OH}^{\circ}\right)[11,12]$. 
Therefore, the accumulation of oxygen free radicals and activation of neutrophils are strongly implicated as important pathophysiological mechanisms mediating myocardial ischemia $[2,13]$. Thus, the site of inflammation is characterized by a high concentration of stimulated neutrophils, which secrete ROS and proteolytic enzymes [14-16].

Complement activation constitutes facet of inflammation, which occurs during ischemia [17-19]. A variety of entities activate complement, including antibodies, membranes of microorganisms, and free radicals [20]. Although it is known that free radicals activate the complement system, the effect of free radicals on complement transcription remains unexplained.

In the present work, we identified that PEP1261 [21] could inhibit the ROS and lysosomal enzymes release from activated neutrophils isolated from acute myocardial infarct rats [1].

\section{Materials and Methods}

This study conforms to the guiding principles of Institutional Animal Ethics Committee (IAEC), Committee for the Purpose of Control and Supervision of Experiments on Animals (CPCSEA), and the Guide for the Care and Use of Laboratory Animals published by the National Institutes of Health (NIH Publication no. 85-23, revised 1996).

2.1. Chemicals. Phorbol 12-myristate 13 acetate (PMA), cytochrome C, superoxide dismutase, phenol red, dextran, Odianisidine hydrochloride, ficoll-histopaque (Sigma 1077), glycerol, hexadecyltrimethylammonium bromide, Hank's balanced salt solution (HBSS), horse radish peroxidase, and Triton X100 were purchased from Sigma (St. Louis, MO, USA). Folin-Ciocalteau reagent, hemoglobin, hydrogen peroxide, and p-nitrophenol phosphate were obtained from Sisco Research Laboratories (Bombay, India). All other chemicals used were of analytical grade.

2.2. Experimental Animals. Female rats (Wistar) weighing 180-200 g were inbred in a pathogen free facility, and they were maintained in environmentally controlled rooms with $12 \mathrm{~h}$ light/dark cycle. The animals received commercial rat diet and water ad libitum.

2.3. Synthesis of PEP1261. PEP 1261 used in this study was synthesized by solution phase methodology as represented schematically in Figure 1 and purified by column chromatography. The homogeneity of the peptide was established by thin layer chromatography. Proton NMR spectra and IR spectra were recorded using Brucker $300 \mathrm{MHz}$ FT-NMR spectrometer and Nicolet DX-20 FT-IR spectrometer, respectively. Amino acid analysis of the peptide derivative was performed by precolumn derivatization with phenylisothiocyanate (PITC) using HPLC. Reverse phase HPLC separation of PTC amino acids was performed with Pharmacia LKB LCC 2252/LKB VWM 2141 unit with fixed or variable wavelength detector at $254 \mathrm{~nm}$. DuPont Zorbax PTH column, $25 \mathrm{~cm}$ in length, $0.46 \mathrm{~cm}$ internal diameter, and $5 \mu \mathrm{m}$ particle size, was used. The FAB mass spectra were recorded on a JEOL SX 102/DA-6000 Mass Spectrometer Data system [21].

2.4. Development of the Infarct Model. Myocardial infarct model was induced in rats as described earlier [1] with modifications to minimize the early mortality rate. Briefly, the animals were given a mucus secretor blocker glycopyrrolate [2 $\mu \mathrm{g} / \mathrm{kg}$.b.wt., i.m) and were anaesthetized with ketamine [50 mg/kg.b.wt., i.p] and diazepam (2.5 mg/kg.b.wt., i.p]. After anesthesia, endotracheal intubation was performed and a tube connected to a positive pressure respirator was introduced. After establishing positive pressure respiration, left intercostal thoracotomy was performed using aseptic technique, and the third and fourth intercostal ribs were separated with a small retractor to expose the heart. The pericardium was opened carefully avoiding any injury to the heart. The left coronary artery (LCA) and its branches could be seen easily without any amplification or use of a surgical microscope. The pattern of the LCA was carefully examined in order to ligate the left anterior descending coronary artery. A 6-0 atraumatic proline silk suture was passed through the epicardial layer around the midway of the left anterior descending coronary artery. Following coronary occlusion, the thorax was closed in layers, the endotracheal tube was removed, and the animals were brought back to normal respiration.

2.5. Isolation of Neutrophils from Rats. Blood was drawn from sham-operated and infarct rats, and neutrophils were separated according to Newman et al. [22]. The neutrophils were suspended in HBSS, and the cell concentration was determined using a haemocytometer.

2.6. Stimulation Studies. To ascertain the optimal concentration of PEP1261 on neutrophils isolated from rats, myeloperoxidase (MPO) was used as a sensitive marker and the results showed an inhibitory concentration $\left(\mathrm{IC}_{50}\right)$ of $94.10 \pm 8.38 \mu \mathrm{M}$ (data not shown). Hence, further studies were carried out with an optimal concentration of $120 \mu \mathrm{M}$ for PEP1261 and KRDS. Neutrophils $\left(1 \times 10^{6}\right.$ cells/well) were left for adherence for $1 \mathrm{~h}$, and they were stimulated by the addition of PMA $(100 \mathrm{ng} / \mathrm{mL})$ [23] for $1 \mathrm{~h}$ at $37^{\circ} \mathrm{C}$. In the case of shamoperated and infarct rat, neutrophils were isolated and after $1 \mathrm{~h}$ adherence and PEP1261 was added. The culture was terminated after $1 \mathrm{~h}$ of PEP1261 treatment.

MPO was assayed in the cell lysate after extracting the enzyme in phosphate buffer containing hexadecyl trimethylammonium bromide [24].

For $\mathrm{H}_{2} \mathrm{O}_{2}$ assay [25], the medium was removed and to the adherent neutrophils, $100 \mu \mathrm{L}(1 \mu \mathrm{g} / \mathrm{mL})$ of PMA was added followed by the addition of $1000 \mu \mathrm{L}$ of HBSS containing horse radish peroxidase (19units/mL) and phenol red $(0.02 \%)$. After $1 \mathrm{~h}$ of incubation, $100 \mu \mathrm{L}$ of $1 \mathrm{M} \mathrm{NaOH}$ was added and the color developed was read at $605 \mathrm{~nm}$.

For the assay of $\mathrm{O}_{2}{ }^{--}[26]$, the medium was removed and the adherent neutrophils were incubated for $1 \mathrm{~h}$ in HBSS containing $80 \mu \mathrm{M}$ cytochrome $\mathrm{C}$ and $10 \mu \mathrm{M}$ PMA. The color developed in the supernatant was read at $580 \mathrm{~nm}$. The amount 


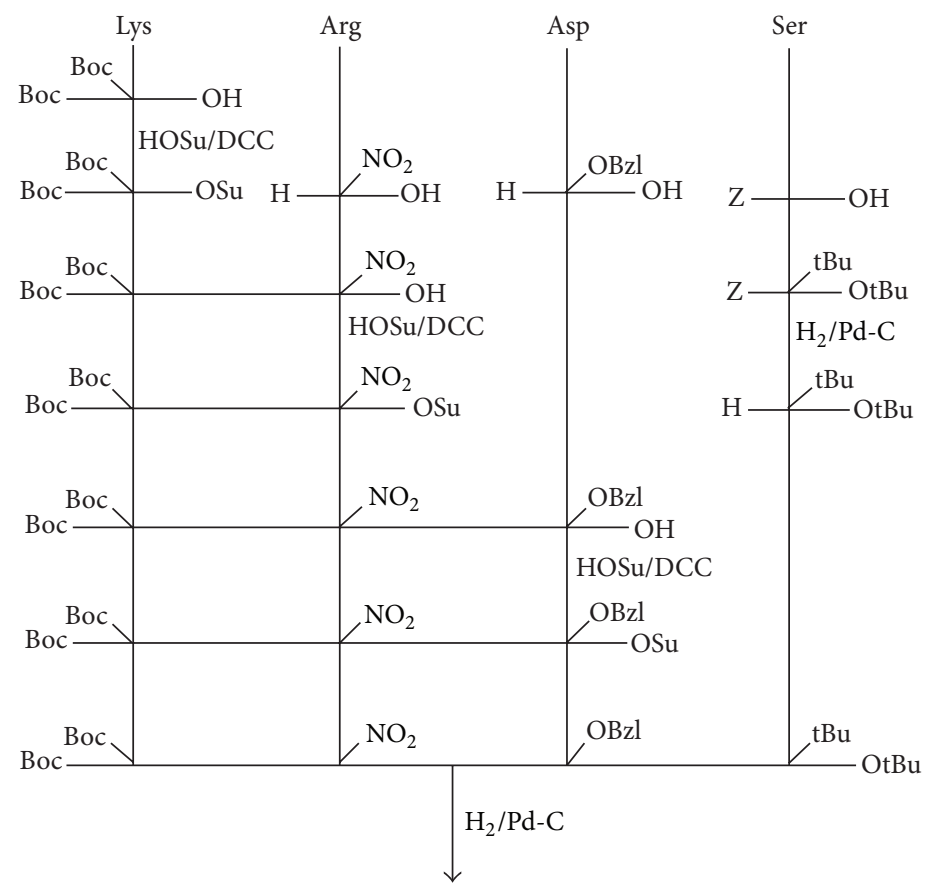

Boc-Lys(Boc)-Arg-Asp-Ser(tBu)-OtBu<smiles>CC(C)(C)OCC(NC(=O)C(CC(=O)[O-])NC(=O)C(CCCNC(=N)N)NC(=O)C(CCCCNC(=O)OC(C)(C)C)NC(=O)OC(C)(C)C)C(=O)OC(C)(C)C</smiles>

FIGURE 1: Scheme of synthesis of PEP1261 with its structure.

of $\mathrm{O}_{2}{ }^{--}$released was measured by the amount of cytochrome $\mathrm{C}$ using a molar extinction coefficient of $21 \times 10^{3} \mathrm{M}^{-1} \mathrm{~cm}^{-1}$.

For the lysosomal enzyme assays, after removing the cell free medium, the cells were lysed by adding $0.5 \mathrm{~mL}$ of ice cold $0.1 \%$ Triton X100 in $0.25 \mathrm{M}$ sucrose, subjected to repeated freezing and thawing and assayed for acid phosphatase [27] and cathepsin D [28].

2.7. Complement Expression Study. Total RNA from neutrophils isolated from sham operated and infarct tissue was extracted using Trizol reagent (kit supplied by Gibco-BRL (Life technologies) (US Patent no. 5,346,94)) a monophasic solution of phenol and guanidine isothiocyanate developed by Chomczynski and Sacchi [29] (Table 1).

2.8. Statistical Studies. Univariate analysis was carried out for all the parameters, and the results were analyzed by nonparametric statistics Mann-Whitney " $U$ ” test.

\section{Results}

Studies on PMA stimulated rat neutrophils showed the beneficial effect of PEP1261 on the ROS generation and lysosomal enzymes release, and, hence, experiments were performed on neutrophils isolated from myocardial ischemic rats. While the levels of $\mathrm{H}_{2} \mathrm{O}_{2}$ and $\mathrm{O}_{2}{ }^{--}$were significantly increased in both PMA stimulated and in the neutrophils isolated from myocardial infarct rats, PEP1261 showed a protective effect towards free radical generation, irrespective of the nature of the stimulant $(P<0.01)$ (Figure 2$)$.

In comparison with PMA treated cells, PEP1261 cells demonstrated a significant decrease in PMA-induced MPO activation $(P<0.01)$. Consistent with these observation, neutrophils derived from myocardial infarct rat were significantly inhibited upon PEP1261 treatment $(P<0.01)$.

Lysosomal enzymes release in response to PMA was also enhanced in neutrophils and was corrected by treatment with PEP1261 as shown in Figure 3. PMA stimulation resulted in a moderate increase $(P<0.05)$ in the levels of cathepsin D and acid phosphatase, and the levels of these enzymes were noticed to decrease upon treatment with PEP1261 $(P<0.05)$.

To ensure that the results obtained are from the neutrophils derived from the acute myocardial infarcted rats, RT-PCR amplification from total RNA extracts was used to establish the presence and relative values of the mRNAs for 
TABLE 1

\begin{tabular}{lccc}
\hline Gene & Sequence of primer (Sense) & Sequence of primer (Antisense) & Product length (base pair) \\
\hline C5 & CAGCATAATTCAGGGTGAACG & CAGCTTGTCATTTGAGCCAC & 315 \\
C6 & TGCAGTGACAAAACGGAACAACCTC & TGCAGTCTTCCTCTTGTCGCTTCTC & 338 \\
C7 & GGAACAGAGTCAATACCAAAAG & ACTGCGTGAAGAAGATGATGAAGAT & 248 \\
C8 & GACTGCGACCCTCTTGACTCTGCTC & TTTCGGAAGGTACTGACAGCCATGG & 258 \\
C9 & GAATGAGCCCCTGGAGTGAATGGTC & CATTTCCGCAGTCATCCTCAGCATC & 316 \\
\hline
\end{tabular}

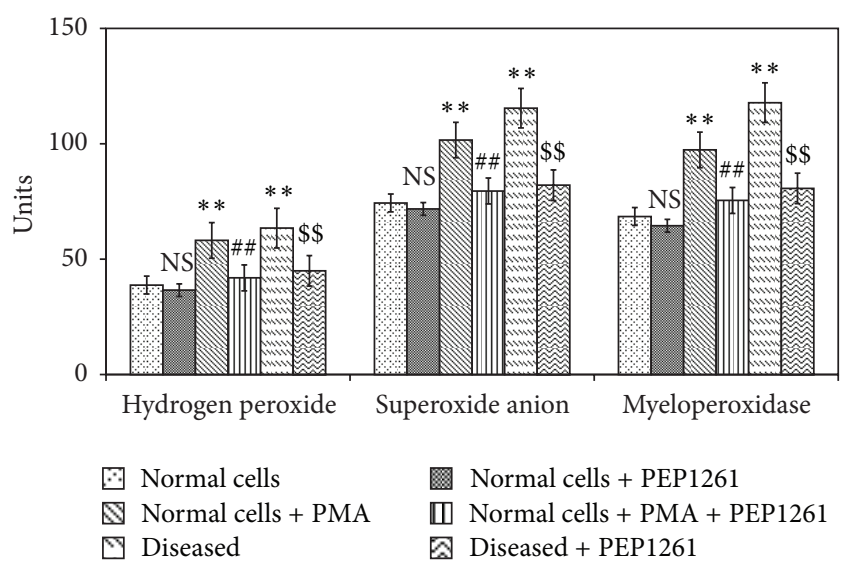

FIGURE 2: Effect of PEP1261 on myocardial infarct rat neutrophils ROS generation. All values are mean $\pm \mathrm{SD}(n=20) . \mathrm{H}_{2} \mathrm{O}_{2}$ level was expressed as $\mu$ moles of $\mathrm{H}_{2} \mathrm{O}_{2}$ liberated $/ 0.5 \times 10^{6}$ cells. $\mathrm{O}_{2}{ }^{\circ-}$ level was expressed as nmoles of $\mathrm{O}_{2}^{-}$liberated $/ \mathrm{min} / \mathrm{mg}$ protein. MPO level was expressed as $\mu$ moles of $\mathrm{H}_{2} \mathrm{O}_{2}$ utilized $/ \mathrm{min} / \mathrm{mg}$ protein. NS = nonsignificant as compared to control; ${ }^{* *} P<0.01$ as compared to control; ${ }^{\# \#} P<0.01$ as compared to PMA stimulated cells; ${ }^{\$ \$} P<0.01$ as compared to rat myocardial infarct cells.

the functional terminal $\mathrm{C}$ proteins, namely, $\mathrm{C} 5, \mathrm{C} 6, \mathrm{C} 7, \mathrm{C} 8$, C9, and GAPDH (Figure 4).

\section{Discussion}

Neutrophils represent the first line of host defense against all types of infection, and they are also involved in the pathology of various inflammatory conditions [30]. The ability to survive an infection challenge might depend on the appropriate modulation of these neutrophil functions [31].

Oxidative stress, defined as an increase in the production of ROS, namely, $\mathrm{O}_{2}{ }^{--}, \mathrm{H}_{2} \mathrm{O}_{2}$, and $\mathrm{OH}^{\bullet}$ has been related to reperfusion injury in heart and other organs [32, 33]. Neutrophils recruitment depends on the presence of inflammatory mediator. These cells, therefore, may exacerbate tissue injury through the release of free radicals and proteolytic enzymes. The oxygen metabolites are produced by a membrane bound enzyme complex, the NADPH oxidase. Thus, in ischemic conditions, there is always the adhesion and activation of neutrophils with the generation of free radicals. Hence, the development of tissue injury depends upon the balance between the generation of ROS and tissue antioxidant status [34]. Any disturbance in this equilibrium in favor of free radicals causes an increase in oxidative stress and initiates

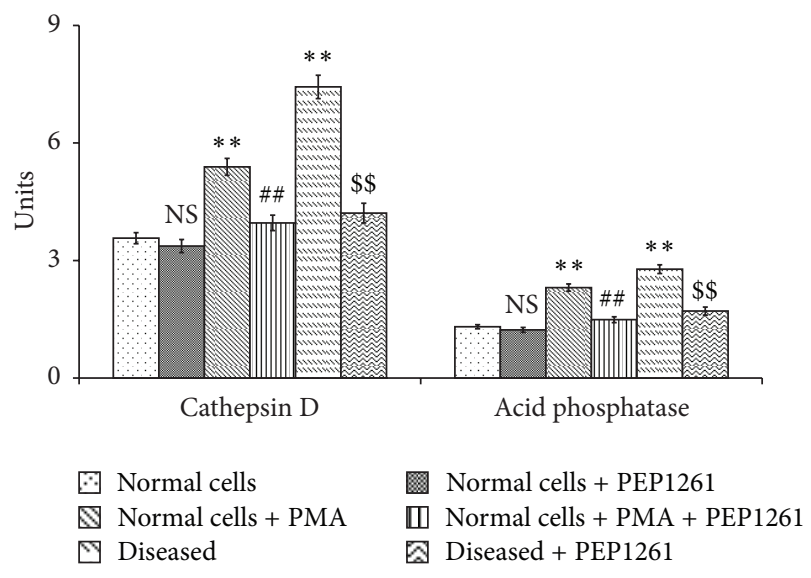

FIGURE 3: Effect of PEP1261 on myocardial infarct rat neutrophils lysosomal release. All values are mean \pm SD $(n=20)$. Acid phosphatase activity was expressed as $\mu$ moles of p-nitrophenol liberated/h/mg protein. Cathepsin D activity was expressed as $\mu$ moles of tyrosine liberated $/ \mathrm{h} / \mathrm{mg}$ protein. NS = nonsignificant as compared to control; ${ }^{*} P<0.05$ as compared to control; ${ }^{\#} P<0.05$ as compared to PMA stimulated cells; ${ }^{\$} P<0.05$ as compared to rat myocardial infarct cells.

subcellular changes leading to cardiomyopathy and heart failure [10].

Several drugs were found to curtail these deleterious effects, and of recent interest is the role of sequence specific peptides as therapeutic agents in many disorders. The sequence H-Lys-Arg-Asp-Ser-OH (KRDS) is an analog of RGDS and corresponds to residues 39-42 of human lactoferrin. It is well known that human lactoferrin exhibits antiinflammatory and antimicrobial properties, and it has a role in regulating various components of the immune system, growth factor activity, and in inhibiting platelet aggregation [35]. This sequence, situated near the N-terminal region of the protein and known to inhibit platelet aggregation in vitro conditions, was suitably modified with hydrophobic groups (PEP1261) to enhance the permeability across the membrane and also to increase the stability.

Preliminary studies were carried out using PMA stimulated human neutrophils, and PEP1261 was found to significantly inhibit ROS species generation and lysosomal enzymes release at a concentration of $120 \mu \mathrm{M}$. The concentration of $120 \mu \mathrm{M}$ did not seem to be very high since earlier experiments showed that the peptide inhibited platelet aggregation only after critical micellar concentration (CMC) $(60 \mu \mathrm{M})$ was 


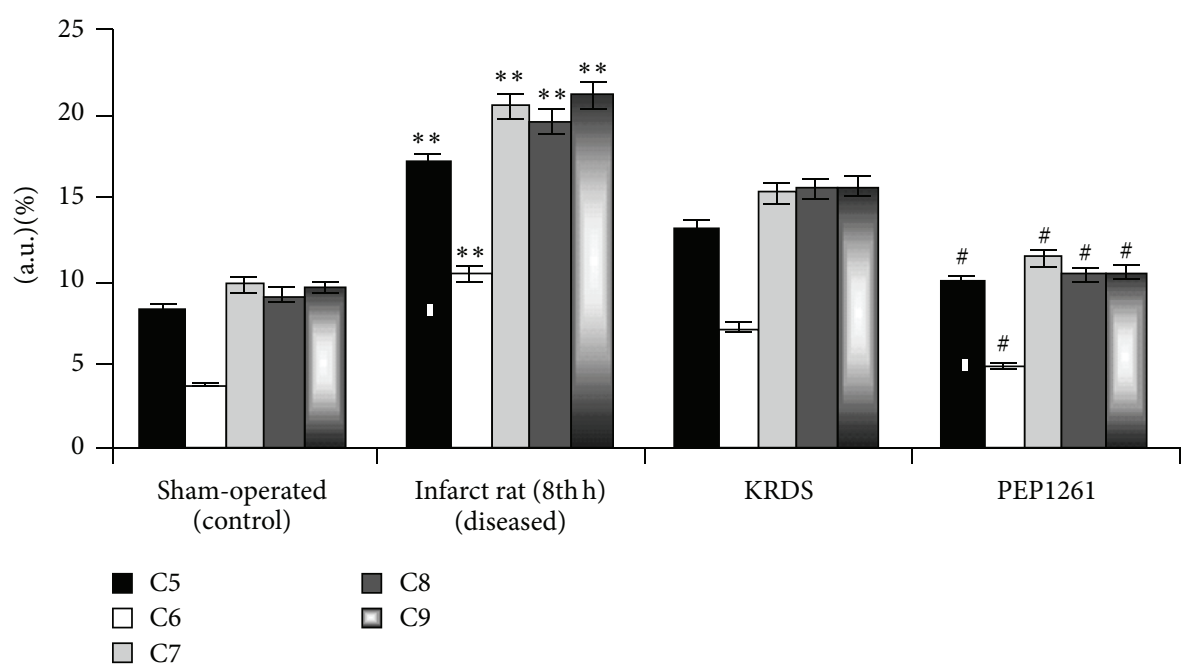

Figure 4: Densitometry analysis of the functional complement factor proteins. All values are mean $\pm \operatorname{SD}(n=6) .{ }^{* *} P<0.01$ as compared to control and ${ }^{\sharp} P<0.05$ as compared to diseased (8th hour infarct myocardium) using post hoc tukey's test.

achieved and a concentration above $153 \mu \mathrm{M}$ was found to effectively inhibit platelet aggregation [21].

MPO activity, a marker of leukocyte accumulation, was markedly elevated in heart injury. It was shown recently that the neutrophils characteristically invaded the myocardial tissue during ischemia and they were observed to be the major source of free radicals [2]. The result of this study showing PEP1261 significantly reducing $\mathrm{O}_{2}{ }^{--}, \mathrm{H}_{2} \mathrm{O}_{2}$, and MPO levels both in the case of stimulated and myocardial rat infarct neutrophils was also in agreement with that of Manikandan et al. [36].

The extensive release of $\mathrm{O}_{2}{ }^{--}$derivatives was probably involved in the pathogenesis of tissue damage following ischemia in stroke and myocardial infarction and in respiratory distress syndrome [35]. Ischemia induced an acute inflammatory response in myocardial tissue with an early phase of neutrophil accumulation, which was accelerated by reperfusion. The rise in $\mathrm{H}_{2} \mathrm{O}_{2}$ level during myocardial ischemia indicated that intensification of oxygen free radical production occurred. This might be the result of hypoxanthine conversion by xanthine oxidase, catecholamine autooxidation, polymorphonuclear neutrophil activation, and/or derangement within mitochondrial electron transfer [37]. In experimental models, interventions that depleted neutrophils or inhibited their function caused a significant reduction in myocardial infarct size [36].

Lysosomal enzymes could completely degrade the components of connective tissue such as collagen, proteinmucopolysaccharide complexes, glycoproteins, and elastin [38]. These observations concurred with our findings that there was an increased activity of lysosomal enzymes such as acid phosphatase and cathepsin D in myocardial ischemic neutrophils which were well brought under the control levels upon PEP1261 treatment.

In summary, neutrophil-mediated ROS, as well as lysosomal enzyme leak in particular, increases after injury. Treatment with PEP1261 attenuates ROS generation and may attenuate cardiac inflammation. We propose that these observations reflect a role for complement mediated activation of neutrophil in acute myocardial infarction. Our observation may suggest novel strategies to attenuate inflammation during cardiac injury and suggest that a better understanding of inflammatory cells and environmental stimuli that alter ROS levels is warranted.

\section{Acknowledgment}

The financial assistance by C.S.I.R., New Delhi to two of the authors, (MS) and (PM) is gratefully acknowledged.

\section{References}

[1] M. Sumitra, P. Manikandan, M. Nayeem et al., "Time course studies on the initiation of complement activation in acute myocardial infarction induced by coronary artery ligation in rats," Molecular and Cellular Biochemistry, vol. 268, no. 1-2, pp. 149-158, 2005.

[2] P. Manikandan, M. Sumitra, M. Nayeem et al., "Time course studies on the functional evaluation of experimental chronic myocardial infarction in rats," Molecular and Cellular Biochemistry, vol. 267, no. 1-2, pp. 47-58, 2004.

[3] D. E. Chambers, D. A. Parks, and G. Patterson, "Xanthine oxidase as a source of free radical damage in myocardial ischemia," Journal of Molecular and Cellular Cardiology, vol. 17, no. 2, pp. 145-152, 1985.

[4] J. M. McCord, "Oxygen-derived free radicals in postischemic tissue injury," New England Journal of Medicine, vol. 312, no. 3, pp. 159-163, 1985.

[5] P. Factor, "Is it the inflammation or the free radicals that cause ischemia/reperfusion lung injury?" Translational Research, vol. 158, no. 1, pp. 14-16, 2011.

[6] S. Hendryk, Z. Czuba, H. Jedrzejewska-Szypułka, P. Bazowski, H. Dolezych, and W. Król, "Increase in activity of neutrophils and proinflammatory mediators in rats following acute and 
prolonged focal cerebral ischemia and reperfusion," Acta Neurochirurgica, vol. 106, pp. 29-35, 2010.

[7] D. E. Shifflett, F. G. Bottone, K. M. Young, A. J. Moeser, S. L. Jones, and A. T. Blikslager, "Neutrophils augment recovery of porcine ischemia-injured ileal mucosa by an IL- $1 \beta$ - and COX2-dependent mechanism," American Journal of Physiology, vol. 287, no. 1, pp. G50-G57, 2004.

[8] O. Ohbayashi and H. Takizawa, "Neutrophils and macrophages related to the pathogenesis and disease development of chronic obstructive pulmonary disease by the inflammatory reaction," Nihon Rinsho, vol. 69, no. 10, pp. 1741-1747, 2011.

[9] Y. Suzuki, S. Ohno, R. Okuyama et al., "Determination of chronic inflammatory states in cancer patients using assay of reactive oxygen species production by neutrophils," Anticancer Research, vol. 32, no. 2, pp. 565-570, 2012.

[10] P. K. Singal, N. Khaper, V. Palace, and D. Kumar, "The role of oxidative stress in the genesis of heart disease," Cardiovascular Research, vol. 40, no. 3, pp. 426-432, 1998.

[11] H. Iwamura, A. R. Moore, and D. A. Willoughby, "Interaction between neutrophil-derived elastase and reactive oxygen species in cartilage degradation," Biochimica et Biophysica Acta, vol. 1156, no. 3, pp. 295-301, 1993.

[12] R. Meera, S. Anand, C. V. Ramesh, and R. Puvanakrishnan, "Inhibition of neutrophil derived lysosomal enzymes and reactive oxygen species by a novel tetrapeptide," Inflammation Research, vol. 48, no. 9, pp. 479-484, 1999.

[13] P. Manikandan, M. Sumitra, S. Aishwarya, B. M. Manohar, B. Lokanadam, and R. Puvanakrishnan, "Curcumin modulates free radical quenching in myocardial ischaemia in rats," The International Journal of Biochemistry \& Cell Biology, vol. 36, no. 10, pp. 1967-1980, 2004.

[14] E. Brandt, J. Van Damme, and H. D. Flad, "Neutrophils can generate their activator neutrophil-activating peptide 2 by proteolytic cleavage of platelet-derived connective tissue-activating peptide III," Cytokine, vol. 3, no. 4, pp. 311-321, 1991.

[15] B. P. Sharonov and I. V. Churilova, "Inactivation and oxidative modification of $\mathrm{Cu}, \mathrm{Zn}$ superoxide dismutase by stimulated neutrophils: the appearance of new catalytically active structures," Biochemical and Biophysical Research Communications, vol. 189, no. 2, pp. 1129-1135, 1992.

[16] B. P. Sharonov and I. V. Churilova, "The mechanism of superoxide dismutase inactivation by stimulated neutrophils," Doklady Akademii nauk SSSR, vol. 322, no. 1, pp. 185-188, 1992.

[17] K. S. Kilgore, G. S. Friedrichs, J. W. Homeister, and B. R. Lucchesi, "The complement system in myocardial ischaemia/ reperfusion injury," Cardiovascular Research, vol. 28, no. 4, pp. 437-444, 1994.

[18] B. R. Lucchesi, "Complement, neutrophils and free radicals: mediators of reperfusion injury," Arzneimittel-Forschung A, vol. 44, no. 3, pp. 420-432, 1994.

[19] J. W. Homeister and B. R. Lucchesi, "Complement activation and inhibition in myocardial ischemia and reperfusion injury," Annual Review of Pharmacology and Toxicology, vol. 34, pp. 1740, 1994.

[20] C. D. Collard, A. Väkevä, C. Büküsoglu et al., "Reoxygenation of hypoxic human umbilical vein endothelial cells activates the classic complement pathway," Circulation, vol. 96, no. 1, pp. 326333, 1997.

[21] C. V. Ramesh, R. Jayakumar, and R. Puvanakrishnan, "A novel surface-active peptide derivative exhibits in vitro inhibition of platelet aggregation," Peptides, vol. 19, no. 10, pp. 1695-1702, 1998.
[22] S. L. Newman, J. E. Henson, and P. M. Henson, "Phagocytosis of senescent neutrophils by human monocyte-derived macrophages and rabbit inflammatory macrophages," Journal of Experimental Medicine, vol. 156, no. 2, pp. 430-442, 1982.

[23] N. G. Gurskaya, L. Diatchenko, A. Chenchik et al., "Equalizing cDNA subtraction based on selective suppression of polymerase chain reaction: cloning of Jurkat cell transcripts induced by phytohemaglutinin and phorbol 12-myristate 13-acetate," Analytical Biochemistry, vol. 240, no. 1, pp. 90-97, 1996.

[24] A. Seekamp, M. S. Mulligan, G. O. Till, and P. A. Ward, "Requirements for neutrophil products and L-arginine in ischemia- reperfusion injury," American Journal of Pathology, vol. 142, no. 4, pp. 1217-1226, 1993.

[25] E. Pick and D. Mizel, "Rapid microassays for the measurement of superoxide and hydrogen peroxide production by macrophages in culture using an automatic enzyme immunoassay reader," Journal of Immunological Methods, vol. 46, no. 2, pp. 211-226, 1981.

[26] L. A. Guthrie, L. C. McPhail, P. M. Henson, and R. B. Johnston, "Priming of neutrophils for enhanced release of oxygen metabolites by bacterial lipopolysaccharide. Evidence for increased activity of the superoxide-producing enzyme," Journal of Experimental Medicine, vol. 160, no. 6, pp. 1656-1671, 1984.

[27] A. J. Barrett and M. F. Heath, "Lysosomal enzymes," in Lysosomes: A Laboratory Handbook, J. T. Dingle, Ed., Elsevier North Holland Biomedical Press, Amsterdam, The Netherlands, 1977.

[28] A. I. Sapolsky, R. D. Altman, and D. S. Howell, "Cathepsin D activity in normal and osteoarthritic human cartilage," Federation Proceedings, vol. 32, no. 4, pp. 1489-1493, 1973.

[29] P. Chomczynski and N. Sacchi, "Single-step method of RNA isolation by acid guanidinium thiocyanate-phenol-chloroform extraction," Analytical Biochemistry, vol. 162, no. 1, pp. 156-159, 1987.

[30] J. A. Smith, "Neutrophils, host defense, and inflammation: a double-edged sword," Journal of Leukocyte Biology, vol. 56, no. 6, pp. 672-686, 1994.

[31] J. Yee and N. V. Christou, "Neutrophil priming by lipopolysaccharide involves heterogeneity in calcium-mediated signal transduction: studies using fluo-3 and flow cytometry," Journal of Immunology, vol. 150, no. 5, pp. 1988-1997, 1993.

[32] J. Wysocka, "The role of polymorphonuclear granulocytes in biomorphology of thrombus in myocardial infarction," Przeglad Lekarski, vol. 52, no. 12, pp. 606-609, 1995.

[33] J. Wysocka, "Polymorphonuclear leukocytes as a potential source of oxygen free radicals in myocardial infarction," Postpy Higieny i Medycyny Doświadczalnej, vol. 49, no. 2, pp. 297-304, 1995.

[34] F. Morel, J. Doussiere, and P. V. Vignais, "The superoxidegenerating oxidase of phagocytic cells. Physiological, molecular and pathological aspects," European Journal of Biochemistry, vol. 201, no. 3, pp. 523-546, 1991.

[35] B. Leveugle, J. Mazurier, D. Legrand, C. Mazurier, J. Montreuil, and G. Spik, "Lactotransferrin binding to its platelet receptor inhibits platelet aggregation," European Journal of Biochemistry, vol. 213, no. 3, pp. 1205-1211, 1993.

[36] P. Manikandan, M. Sumitra, D. A. Kumar et al., "Antioxidant potential of a novel tetrapeptide derivative in isoproterenolinduced myocardial necrosis in rats," Pharmacology, vol. 65, no. 2, pp. 103-109, 2002.

[37] W. F. Saavedra, N. Paolocci, M. E. S. John et al., "Imbalance between xanthine oxidase and nitric oxide synthase signaling 
pathways underlies mechanoenergetic uncoupling in the failing heart," Circulation Research, vol. 90, no. 3, pp. 297-304, 2002.

[38] L. V. Ravichandran, R. Puvanakrishnan, and K. T. Joseph, "Alterations in the heart lyososomal stability in isoproterenol induced myocardial infarction in rats," Biochemistry International, vol. 22, no. 2, pp. 387-396, 1990. 


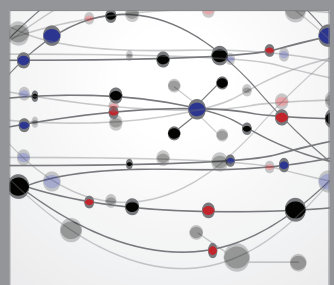

The Scientific World Journal
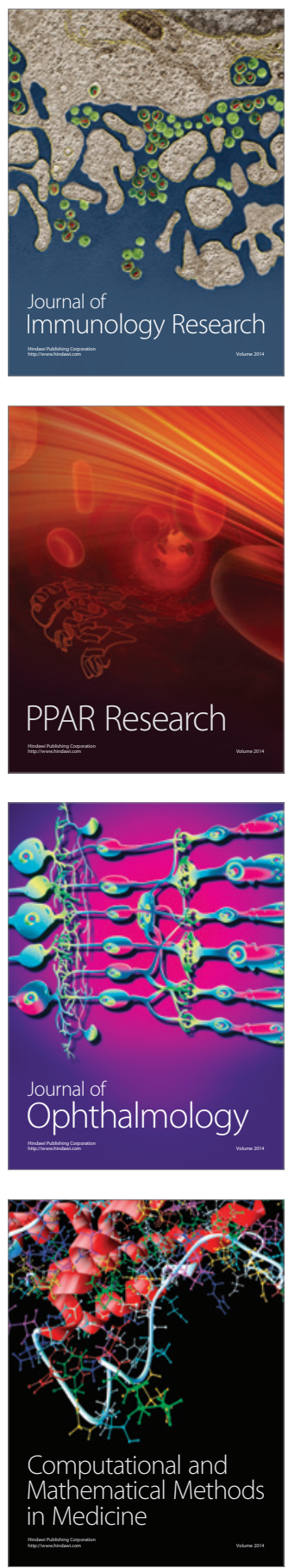

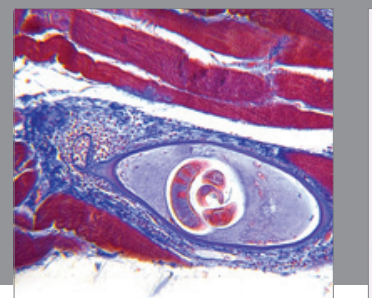

Gastroenterology

Research and Practice
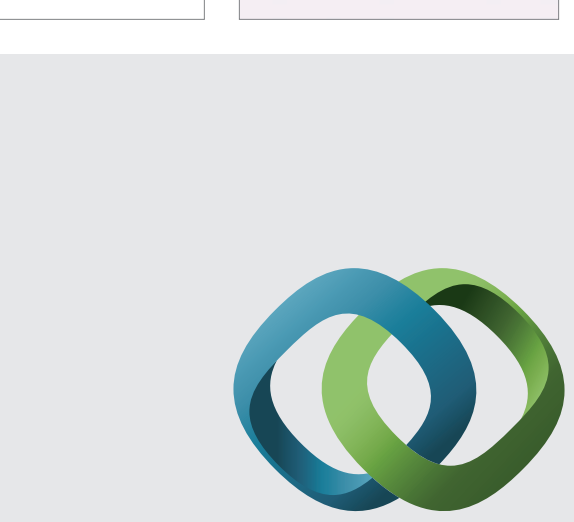

\section{Hindawi}

Submit your manuscripts at

http://www.hindawi.com
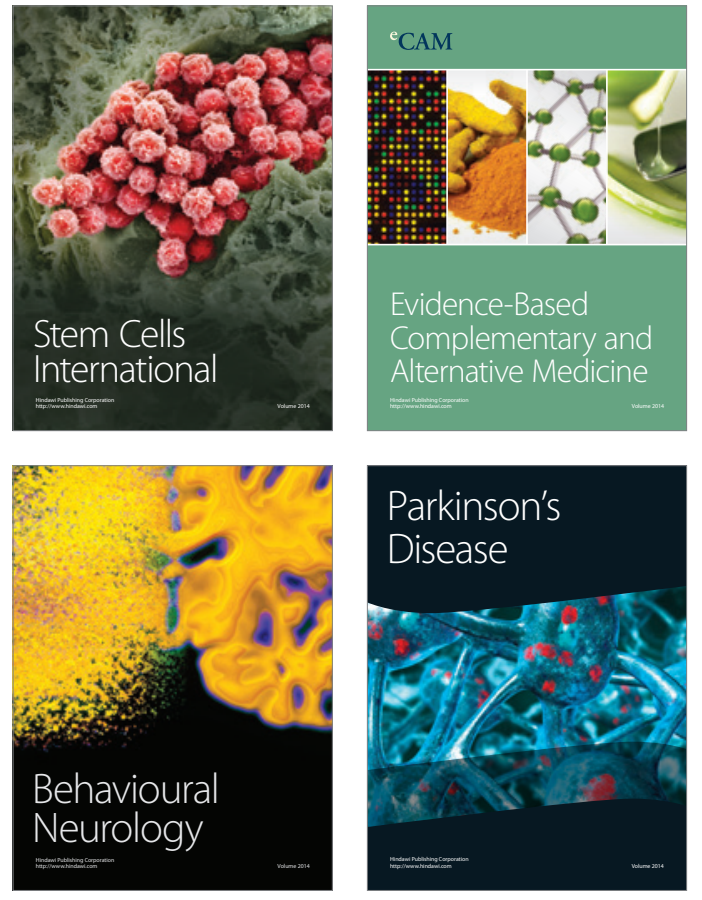
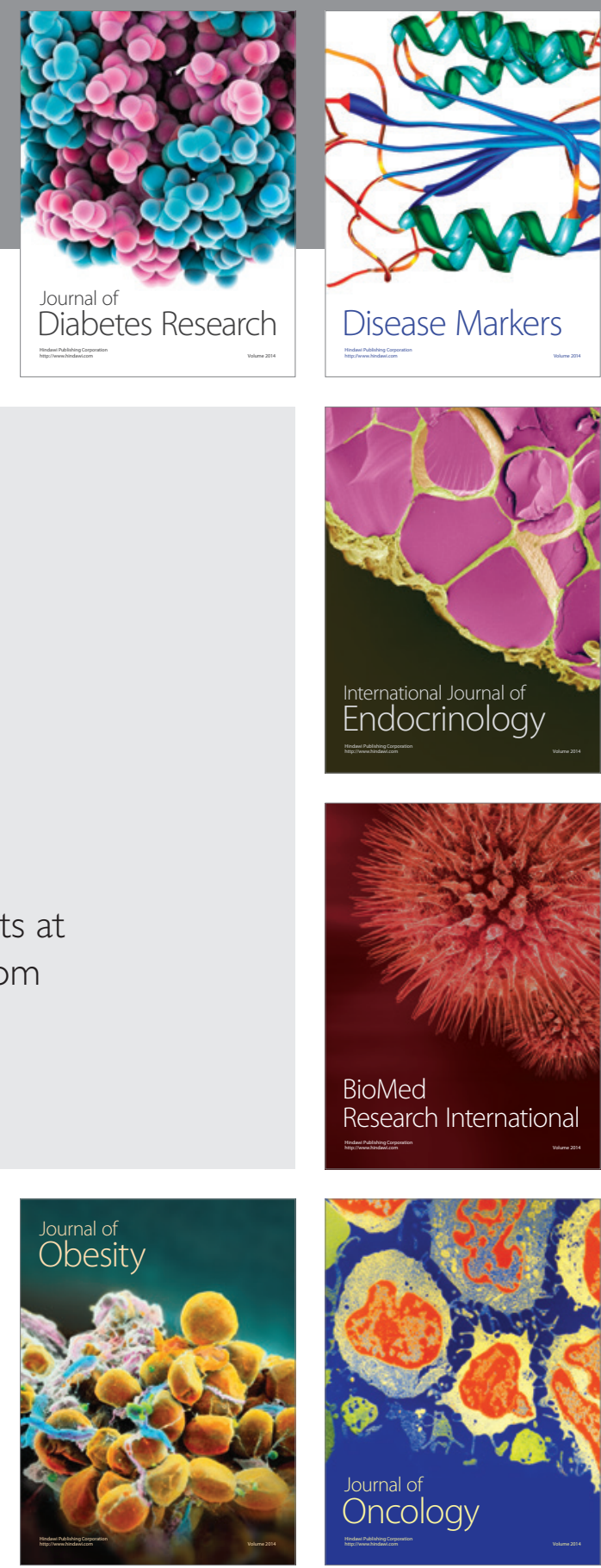

Disease Markers
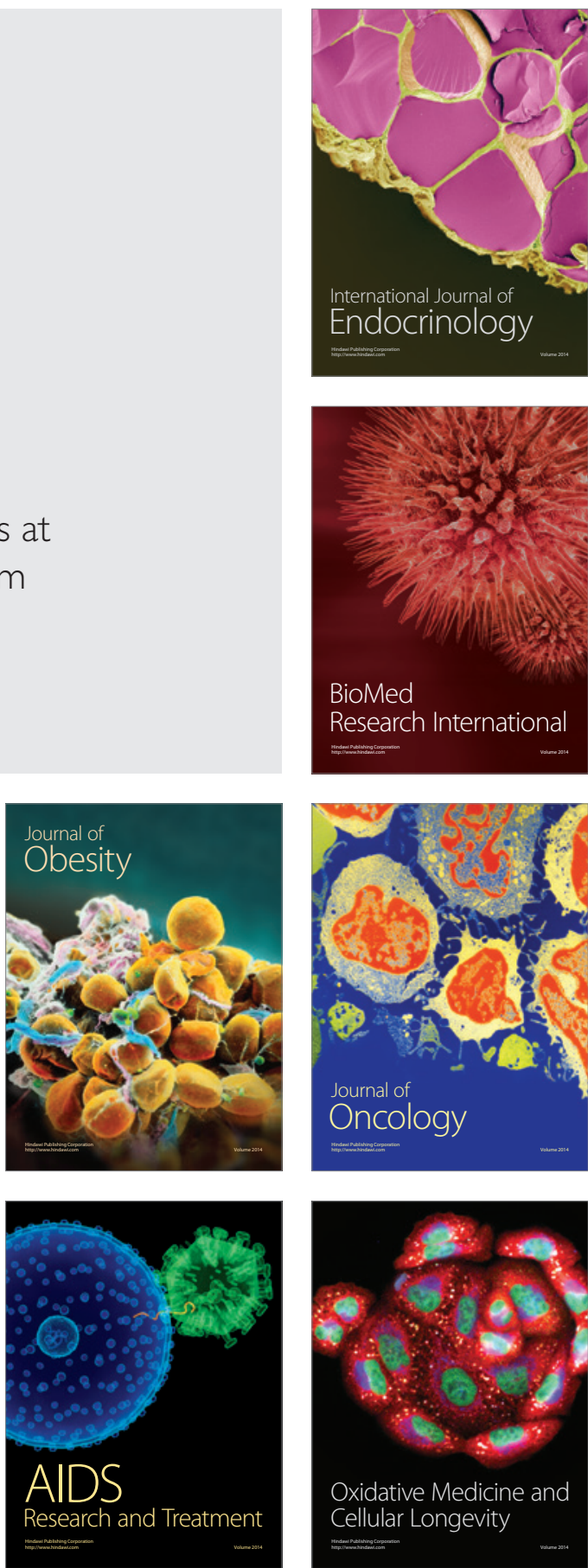